ORIGINAL PAPER

Eur. J. Histochem

45: 239-248, 2001

(C) Luigi Ponzio e figlio - Editori in Pavia

\title{
Comparative analysis of NADPH-diaphorase positive neurons in the rat, rab- bit and pheasant thoracic spinal cord. A histochemical study
}

\author{
D. Kluchová, S. Rybárová, M. Miklošová, K. Lovásová, K. Schmidtová, and F. Dorko
}

Department of Anatomy, Faculty of Medicine, P. J. Šafarik University, Šrobárova 2, 04001 Košice, Slovak Republic

Accepted: 23/2/01

Key words: NADPH-d, distribution, spinal cord, histochemistry

\section{SUMMARY}

The distribution of NADPH-diaphorase (NADPHd) activity was investigated and compared in the rat, rabbit and pheasant thoracic spinal cord. The investigation of all spinal cord regions (laminae) in three experimental species revealed marked differences in the distribution of NADPH-d activity.

Cross sectional analysis of the spinal cord of the rat, rabbit and pheasant confirmed differences in the shape of the gray matter in all examined species. More detailed investigation of Rexed's laminas showed similar distribution of NADPH-d activity in the spinal cord of the rat and rabbit, which were different when compared with the spinal cord of the pheasant.

Ventral horn of the rat and rabbit showed no labelling whereas in pheasant this area possessed a number of scattered, intensively stained neurons. In the location of autonomic preganglionic neurons, differences were found as well. In the rat there was seen a number of densely packed, clearly dark blue coloured neurons. Similarly, these neurons were present in the rabbit spinal cord but they were less numerous. No staining was found in this region of pheasant. Pericentral area (lamina $X$ ) and intermediate zone (laminaVII) revealed the presence of NADPH-d positive neurons in all examined species although they differed in number and shape of their bodies. The dorsal horn showed the presence of NADPH-d staining in all three animals but its distribution was different in medio - lateral direction.

It can be suggested that observed differencies in the presence and distribution of NADPH-d activity across the examined species may reflect different fylogenetic development.

\section{INTRODUCTION}

The nicotinamide adenine dinucleotide hydrogen phosphate - diaphorase (NADPH-d) histochemical reaction involves the transfer of hydrogen from the substrate, NADPH, to a hydrogen acceptor, a tetrazolium salt, which is converted to the insoluble blue formazan (Mizukawa 1990, Scherer-Singler et al., 1983).

The significance of NADPH-d has been elucidated by the discovery that NADPH-diaphorase so closely resembles nitric oxide synthase (NOS) that they may be identical (Hope et al., 1991; Dawson et al., 1991). NOS is the enzyme responsible for the synthesis of nitric oxide (NO), which was accepted as a novel transcellular messenger that is directly involved in a wide range of physiological 
activities (Kiechle and Malinsky, 1993). There is an evidence that NO plays a major role in at least three systems: white blood cells, where it mediates tumoricidal and bactericidal effects; blood vessels, where it represents endothelium-derived relaxing factor activity; neurons, where it functions very much like that of a neurotransmitter (Bredt and Snyder, 1992).

As NO is a gaseous, free radical with a half-life of 5 seconds, it is readily diffusible in tissue (Kiechle and Malinsky, 1993). The detection of its presence is focused upon the enzyme NOS. Previous studies (Hope et al., 1991; Dawson et al., 1991) have demonstrated that endothelial and neuronal forms of NOS are colocalized with NADPH-d in tissue fixed by $4 \%$ paraformaldehyde. This enzyme has a very wide distribution in the brain (Bredt and Snyder, 1990), comparable to the distribution of the major neurotransmitters glutamate and GABA, suggesting an involvement in a large number of different neuronal tissue functions (Garthwaite, 1995). Histochemical characterization of neuronal NADPH was studied more in detail by Hope and Vincent (1989). In the light of the importance of NO, NADPHdiaphorase histochemistry has become the most widely used method to study the distribution of neuronal structures containing NOS.

Our interest was focused on the presence of NADPH-d in the spinal cord of rat, rabbit and pheasant. From previous reports it has been known that NADPH-d is distributed in various segments throughout the entire rat spinal cord (Valtschanoff et al., 1992) as is the distribution of NOS (Saito et al., 1994). Localization of NADPH-d in the spinal cord of cat and dog was studied by Vizzard et al. (1994; 1997). Besides mammalian material, the presence od NADPH-d positivity was studied also in non-mammalian spinal cord cells, e.g. in snake (Jiang and Terashima, 1996), frog (Muňoz et al., 1996) and fish (Funakoshi et al., 1995). Special interest was given to NADPH-d positivity in a subpopulation of autonomic preganglionic neurons (Anderson et al., 1993). Nitrergic autonomic neurons in the rat spinal cord were studied also by Blottner et al., (1993). There is support for the presence of NOS containing neurons in the intermediolateral (IML) cell column of the rat spinal cord (Saito et al., 1994; Dun et al., 1992). This kind of neuron was confirmed in the thoracic spinal cord of rat by combination of histochem- istry, immunochemistry and retrograde labelling (Blottner and Baumgarten, 1992). Histochemistry of NADPH-d as a marker for NOS was used to identify NO synthesizing neurons in peripheral nervous system as well (Grozdanovic et al., 1992, Gulati et al., 1995).

To address the question of whether there are species differencies in the distribution of NOSreleasing neurons in the spinal cord, NOS immunoreactivity was examined in the rat, mouse, cat and squirrel monkey (Dun et al., 1993). This study showed that NOS-positive neurons of spinal cord were similarly distributed across the four species.

Results mentioned above raised our interest on possible species differencies in the distribution of spinal cord NADPH-d positivity in cells of mammalian and non-mammalian material. Choice for the non-mammalian species was birds (Necker, 1999). Since we have not yet found in literature the solution of this problem in birds, the present work aims to first study the localization of NADPH-d neurons and fibres in pheasant spinal cord and to compare its distribution with the distribution of NADPH-d in the rat and rabbit spinal cord.

\section{MATERIALS AND METHODS}

\section{Animal treatment and tissue preparation}

Five adult rats, seven rabbits and four pheasants of both sexes were used in this study. Experimental animals were anesthesized with pentobarbital (30 $\mathrm{mg} / \mathrm{kg}$, i.v.) and immediately killed via intracardiac perfusion with saline followed by $4 \%$ paraformaldehyde $+0.1 \%$ glutaraldehyde buffered with $0.1 \mathrm{M}$ sodium phosphate, $\mathrm{pH}-7.4$. The fixatives were freshly made up immediately prior to perfusion.

Following perfusion, the spinal cord was carefully dissected out and stored in the same fixative for 3-4 hours. Then it was placed in ascending concentrations of sucrose (15-30\%) in the same phosphate buffer for cryoprotection and stored overnight at $4^{\circ} \mathrm{C}$. Spinal cord segments were sectioned transverselly in a freezing microtome at a thickness of $45 \mu \mathrm{m}$.

\section{Histochemical procedure}

NADPH-d histochemical detection was performed as reported in our previous studies 
(Maršala et al., 1997; Kluchová et al., 1999, 2000). Sections were incubated for an hour at $37^{\circ} \mathrm{C}$ in a solution of $1.5 \mathrm{mM}$ nitroblue tetrazolium (NBT, Sigma Chemicals, N-6876), $1.0 \mathrm{mM} \mathrm{B-}$ nicotinamide adenine dinucleotide phosphate (NADPH, Sigma Chemicals, N-1630), 0.5\% Triton $\mathrm{X}-100$ dissolved in $0.1 \mathrm{M}$ phosphate buffer $(\mathrm{pH} 7.4), 10.0 \mathrm{mM}$ monosodium malate (malic acid, Sigma Chemicals, M-1125).

Control sections were treated in the same way but without NADPH in the reaction medium. This was to test for endogenous reduction activity in the corresponding blue formazan product (Hope and Vincent, 1989).

Following the reaction, sections were rinsed in $0.1 \mathrm{M}$ phosphate buffer ( $\mathrm{pH} 7.4$ ), mounted on slides, air-dried overnight and coverslipped with Entellan.

\section{RESULTS}

The investigation of the rat thoracic spinal cord revealed the presence of NADPH-d positive neurons in three main localities: superficial layers of the dorsal horn, pericentral zone and the area of intermediolateral (IML) column (Fig. 1a). The occurence and number of these neurons varied in the spinal cord throughout its entire length. No NADPH-d activity was found in the ventral horn of the rat spinal cord. Differences seen in transverse sections of the spinal cord of the rat and rabbit were noticable only in the shape of the gray matter (Fig. 1a, 1b), but the distribution of NADPH-d positive structures was very similar in both examined species.

On the contrary, pheasant spinal cord showed, in the transverse section, quite a different picture not only in the shape and unclear diffuse border of its gray matter but also in the lack of NADPH-d staining in the IML area (Fig. 1c).

\section{Laminar distribution of NADPH-d activity}

Detailed observation of individual spinal cord layers (laminae) revealed considerable differences in the distribution of NADPH-d staining between mammalian (rat and rabbit) species and non-mammalian (pheasant) species.

Dorsal horn (lamina I-III)

In the dorsal horn of the rat, especially in lamina I and III, NADPH-d presence was seen as a bilaminar staining, with the absence of labelling in lamina II (Fig. 1d). Spinal cord of the rabbit did not show this picture; laminar arrangement was quite diffuse but NADPH-d positivity was found to be interrupted in the dorso-ventral direction by the entering afferent fibers (Fig. 1e). In spite of this, pheasant spinal cord showed complete transfer of NADPH-d labelling from the medial to its lateral part of the dorsal horn in superficial layers (Fig. 1f).

\section{Pericentral region (lamina $\mathrm{X}$ )}

A substantial number of intensively stained NADPH-d positive neurons were located around the central canal (lamina X) of the rat (Fig. 2a). Their fibers were widely branching and intercommunicating, often extending to the dorsal commissure (Fig. 2b). In the rabbit, pericentral neurons were arranged in similar fashion but they were found less numerous than in the rat, often localized closely to the subependymal layer (Fig. 2c). Surprising was the extensive diameter of the rabbit central canal, the largest of all examined animals. Higher magnification of positively stained neurons showed less branching of their processes in comparison with the rat (Fig. 2d). In pheasant pericentral region, considerable differencies were noticed (Fig. 2e). The small, narrow central canal was located much more ventrally than in the rat and rabbit and NADPH-d neurons were seen to be placed more in a dorsal direction with a larger distance from the central canal. The shape of these neurons was different as well, their bodies were smaller in size, possessing only a small number of short, non-branching nerve fibers (Fig. 2f).

\section{Intermediolateral cell column (lamina VII)}

At the margin of the gray matter and the lateral funiculus, intensive NADPH-d staining was found in the rat and rabbit spinal cord (Fig. 1a, b). Clusters of neurons, densely packed, were observed in this area, typical for the presence of sympathetic preganglionic neurons. NADPH-d staining in the IML region of the pheasant was absent (Fig. 1c), but some positively labelled cells were seen in lamina VII located medial to the IML column.

\section{Ventral horn (lamina VIII-IX)}

Both in the rat and rabbit spinal cord, cellular staining for NADPH-d activity in the ventral horn was generally absent (Fig. 3a, b). Pheasant spinal 
Imp. Kluchova 11-02-2002 9:10 Pagina 242
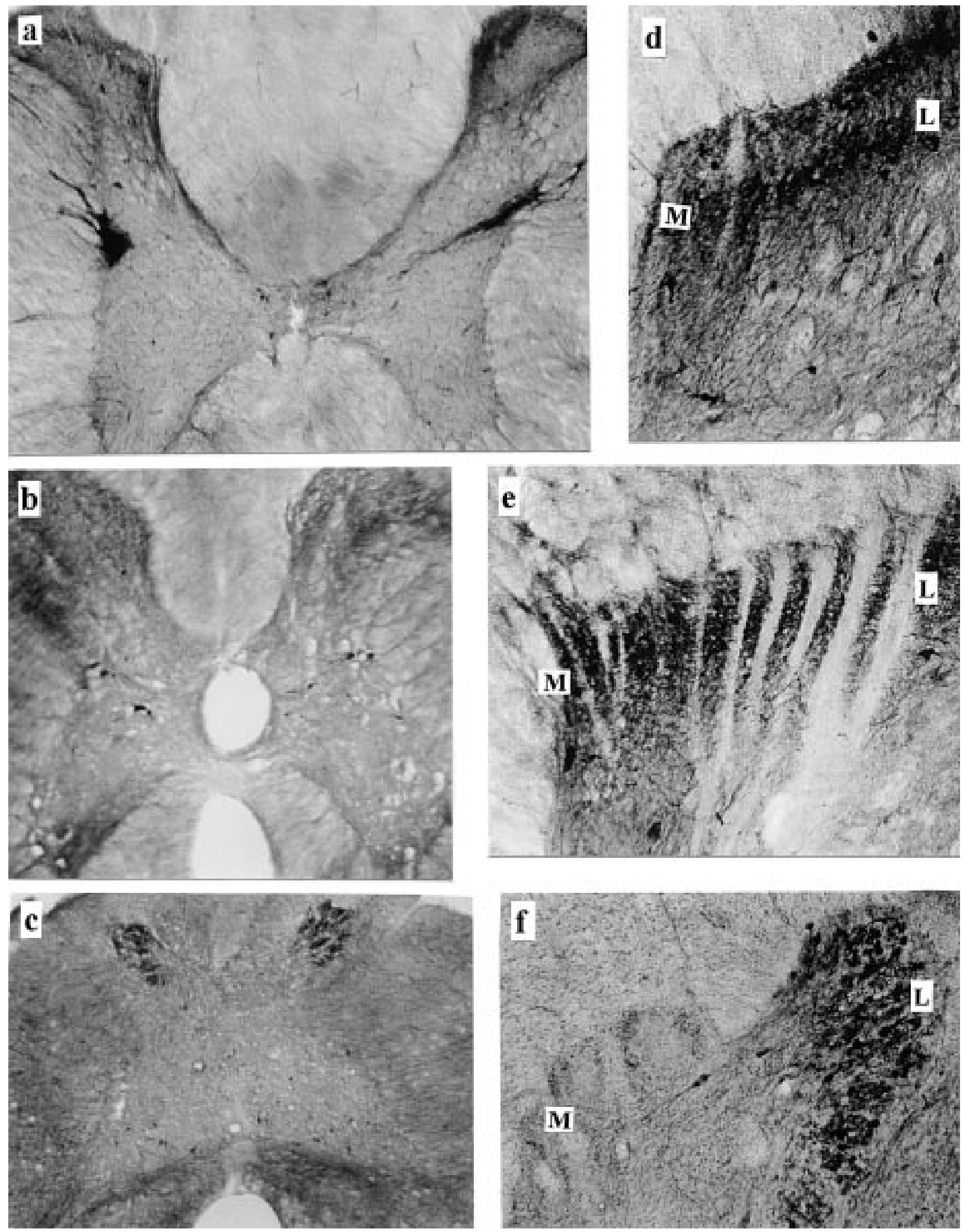
cord was different in this area as well (Fig. 3c). Clearly stained NADPH-d neurons were seen scattered throughout the area of the ventral horn in the thoracic part.

\section{DISCUSSION}

In the present study, the distribution of NADPH-d staining in the spinal cord of three species was examined. The aim of this work was to compare the presence of positively stained neurons and to suggest possible physiological functions of NO in this part of the CNS. It is well known that NO diffuses unhindered from its cells of origin with the potential to affect all cells within its immediate vicinity (Bredt and Snyder, 1992). This property allows NO to play a number of different roles in the CNS where it is regarded as a novel transcellular messenger. As mentioned before, NO generated by nitrergic neurons can be identified by NADPH-d histochemistry (Dawson et al., 1991; Hope et al., 1991). However, caution must be taken in interpretation of these results in the light of recent evidence suggesting that NADPH-d might be only a form of NOS (Schmidt et al., 1992). Furthermore, Matsumoto et al. (1993) have suggested that most of the NADPH-d activity in intact cells is in the particulate fraction and is not NOS. Thus, a one to one correlation between NADPH-d and NOS does not always exist and NOS cells may represent a subpopulation of NADPH-d neurons. In recent studies, NADPH-d histochemistry is considered to be a selective marker for neurons that may synthesize NO both in the spinal cord (Muňoz et al., 1996) and in the brain (Němcová et al., 2000) when describing one species. Most studies carried out point to a co-expression of NADPH$\mathrm{d}$ and NOS enzymes in the same regions (Dawson et al., 1991; Vizzard et al., 1994). Therefore, although the controversy is still open, the use of NADPH-d histochemistry seems to be a powerful procedure to give insight into the organization of NO systems in the spinal cord.
The distribution of NADPH-d and NOS positive neurons in the spinal cord of rat was already described by others (Valtchanoff et al., 1992; Saito et al., 1994). The rabbit spinal cord was examined for the localization of NADPH-d activity as previously published (Kluchová et al., 1999, 2000; Maršala et al., 1997). Both rat and rabbit spinal cords are known to possess five areas which contain NADPH-d positive neurons, i.e. 1/autonomic preganglionic neurons (lamina VII), 2/neurons of the intermediate zone (lamina VII), 3/pericentral neurons (lamina X), 4/neurons in the deep (lamina IV-VI) and 5/superficial (lamina I-III) layers of the dorsal horn. A large number of NADPH-d positive neurons appear to be involved in spinal sensory processes and visceral regulation. The distribution of NADPH-d activity in the pheasant spinal cord was rather different from the above mentioned animals. It was found in areas of: 1 /somatomotor neurons of the ventral horn (lamina VIII-IX), 2/neurons of the intermediate zone (lamina VII), 3/pericentral neurons (lamina $\mathrm{X}$ ), 4/neurons in the deep (lamina IV-VI) and 5/superficial (lamina I-III) part of the dorsal horn. This finding shows clear difference in the presence of NADPH-d between rat and rabbit spinal cord on the one hand and pheasant spinal cord on the other. The omission of NADPH-d staining in autonomic preganglionic neurons and its presence in the ventral horn are the most noticable differences in pheasant spinal cord in comparison with those of the rat and rabbit.

In studies on NADPH-d labelled autonomic neurons of the spinal cord, some species differences have been shown. Positively stained neurons were noticed in IML cell column of the rat (Anderson et al., 1993; Valtschanoff et al., 1992, Blottner and Baumgarten, 1992), but not in the cat (Mizukawa et al., 1989). On the contrary, prominent NADPH-d activity in the IML column of the cat was found by Vizzard et al. (1994). The reason for the difference in IML staining in these two experiments might reflect tissue condition (Mizukawa et al., 1989) or

Fig. 1 - (a)Transverse section through the thoracic spinal cord of the rat shows intensively stained area of autonomic preganglionic neurons. (b)View of the rabbits spinal cord reveals solitary arranged NADPH-d positive neurons in the IML area. (c)Note different shape and unclear border of gray matter in the section though the spinal cord of pheasant. No staining can be seen in the area of IML column. (d)Bilaminar NADPH-d staining in the superficial layers in left dorsal horn of the rat. (M - medial, L lateral). (e)NADPH-d positivity in the dorsal horn of the rabbit is interrupted by the afferent nerve fibres. (M - medial, L - lateral). (f)No staining in the medial part and positive NADPH-d reaction in the lateral direction were seen in neurons of the pheasants left dorsal horn. (M - medial, L - lateral). 

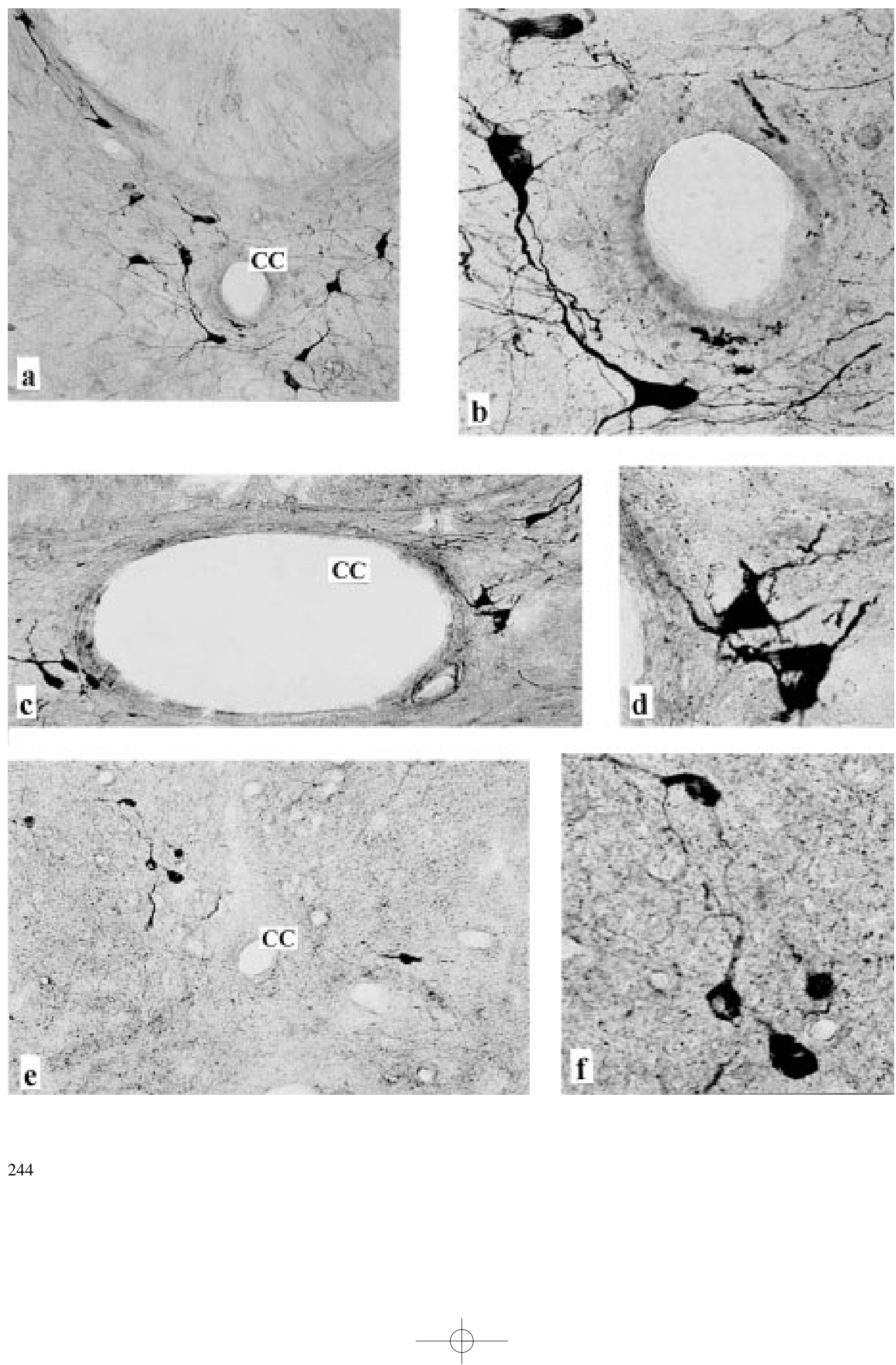
fixation procedure. Autonomic neurons of the sacral parasympathetic nucleus (SPN) also differ across species. In the dog, NADPH-d staining was not seen in this region (Vizzard et al., 1997). A similar situation exists in the cat (Vizzard et al., 1994), whereas in the rat (Valtschanoff et al., 1992) and rabbit (Maršala et al., 1997) the NADPH-d activity was prominent in the region of lumbosacral parasympathetic nucleus.

The results obtained in the present study confirmed the presence of NADPH-d positive neurons in the sympathetic preganglionic neurons of the rat and rabbit, but not in the pheasant spinal cord. Some NADPH-d positive cells were also located medial to the IML horn in the region corresponding to the intercalated and dorsal commissural nuclei, which are known to contain sympathetic preganglionic neurons. NOS-immunoreactive cells were observed in this region of other species, too (Vizzard et al., 1994). It is clear from these findings that $\mathrm{NO}$ can function as a neurotransmitter in sympathetic preganglionic neurons of the pheasant and is involved in spinal cord visceral regulation.

In the ventral horn of the thoracic spinal cord of the rat and rabbit, there were not found any NADPH-d positive neurons. As previously descibed by others, this phenomenon is common to other animals in physiological conditions (Valtschanoff et al., 1992; Dun et al., 1993). Different results were obtained after rhizotomy (Vizzard et al., 1993) and in injured neurons after axonal damage when motoneurons were capable of expressing NADPH-d and NOS activity (Wu, 1993).

Despite the general lack of staining of motoneurons in the spinal cord of the cat and the rat, in some cases NADPH-d and NOS positive neurons were seen in this region (Vizzard et al., 1994). In the cervical spinal cord of the cat these neurons were located in the ventrolateral gray matter. This location suggests that these neurons may be a part of the spinal accessory nucleus. Results obtained by us in pheasant confirmed the presence of NADPH-d positive neurons scattered throughout the area of the ventral horn. The small number of
NADPH-d and NOS positive neurons in the region of the ventral horn raises the possibility that: $1 /$ there is a population of motoneurons with unusual neurochemical properties, or 2/there are present other types of neurons (e.g., autonomic or afferent) in addition to motoneurons (Vizzard et al., 1994). It seems likely that positively stained cells in this region are autonomic cells, or an unusual group of motoneurons. In the study of Pullen et al., (1997), some sections of thoracic spinal cord of the cat exhibited distinct medial and lateral groups of motor neurons showing NOS staining. Retrograde labelling experiments revealed that cat thoracic motor neurons innervating external intercostal muscles are located along with levator costae motor neurons as a group in the ventrolateral tip of the ventral horn, whereas motor neurons projecting to the internal intercostal nerve are sited more dorsomedially (Johnson, 1986). It may be speculated that NADPH-d positivity in pheasant thoracic motoneurons reflects their different function because of the presence of wings in birds. Another possibility to explain this issue is that birds are known to possess much more propriospinal connections compared to other species (Maršala, 1985). Further studies will be needed to confirm some of these hypotheses.

High activity of NADPH-d was noted in the dorsal horn of all three species especially in lamina I and II, but the distribution of this activity was quite different. In the rat, the dorsal horn staining consisted of two distinct bands separated by a prominent unstained region. This banding was not observed in the rabbit; here the NADPH-d activity was distributed throughout lamina I and II in a more diffuse manner. The difference in the pheasant dorsal horn was seen mostly in the mediolateral distribution of NADPH-d positivity. Strongly stained structures in the lateral part of superficial layers of the dorsal horn was in marked contrast with the no staining in its medial part. The fact that afferent fibers, before entering the dorsal horn, are divided into medial and lateral fascicles (Maršala, 1985), could contribute to an explanation of this

Fig. 2 - (a)Numerous NADPH-d positive neurons around the central canal of the rat. (CC - central canal). (b)Higher magnification of pericentral neurons shown in Fig. 2a. (c)Note the extensive diameter of the rabbits central canal. (CC - central canal). (d)Detailed view of neurons shown in Fig. 2c. Their location was closer to subendymal layer. (e)Pericentral area of pheasant spinal cord. Neurons were more distant to the central canal. (CC - central canal). (f)Less stained pericentral neurons shown in Fig. 2e. revealed in higher magnification only a small number of short, non-branching nerve fibers. (CC - central canal). 

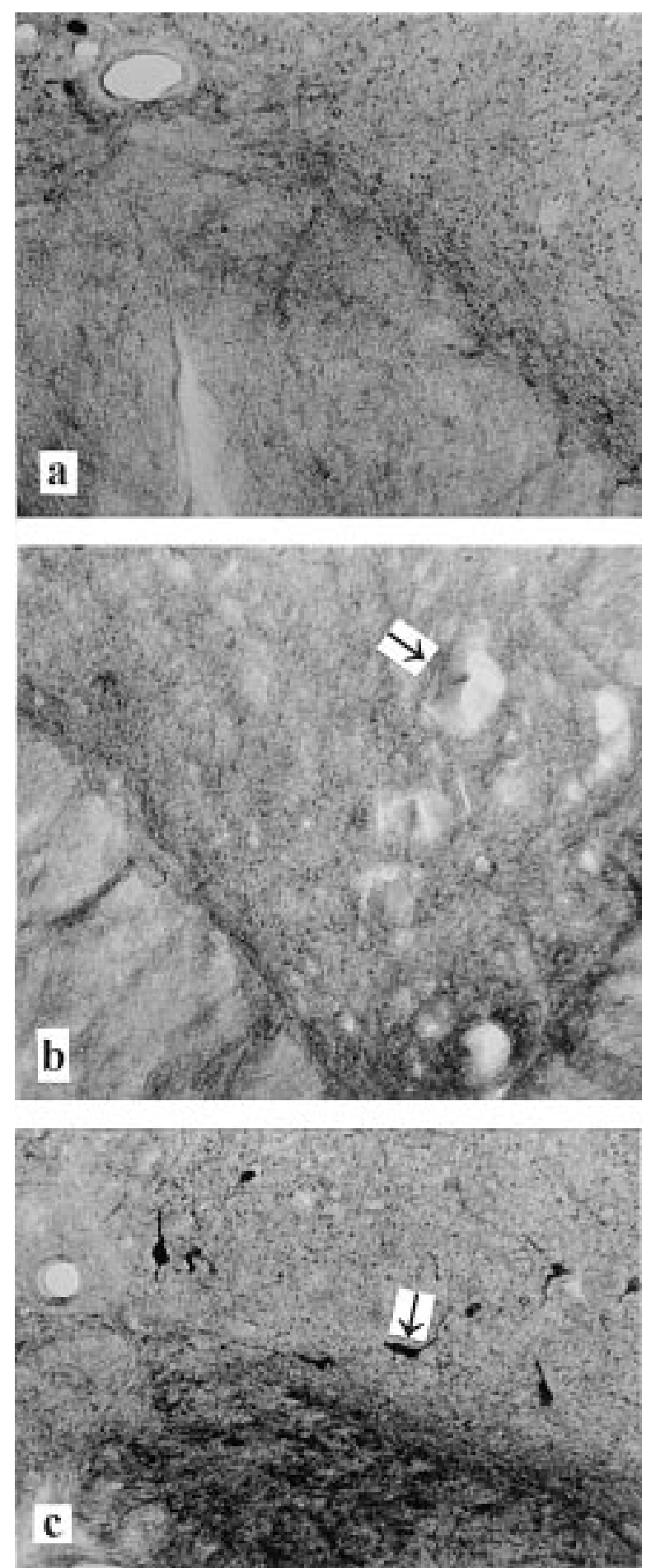

Fig. 3 - (a)No NADPH-d positive or negative cells were seen in the ventral horn of the rat. (b)Neurons of the rabbit ventra horn were visible in shape (arrow) but NADPH-d negative. (c)NADPH-d positive neurons (arrow) were seen scattered throughout the ventral horn of the pheasant. finding. The medial fascicle consists of thick myelinated fibers which are connected in the periphery to such structures as the Golgi neurotendinous organ, neuromuscular spindles and Meissner's and Paccini's corpuscules. Therefore, these fibers conduct information from specialized sensory receptors. The lateral part of the afferent fibers carries information from mechanical, thermal and pain receptors ( Maršala, 1985). The fact that neurons of both parts of the dorsal horn possess different chemical substances may reflect different function of these neurons in the medial and lateral parts of the dorsal horn (O’Brien et al., 1989).

Differences in afferent projections and NADPH$\mathrm{d}$ staining were also noticed in the cat. For example, pelvic visceral afferents, and to a lesser extent VIP-containing afferents, project around the medial side of the dorsal horn to the dorsal commissure (Kawatani et al., 1985). This medial pathway did not exhibit NADPH-d activity, suggesting that NADPH-d is present in only a subpopulation of VIP-ergic visceral neurons.

From our observations, it may be suggested that nerve impulse transmission from specialized sensory receptors is accomplished probably by using other neurotransmitters, but not by using NO. In contrast, NO seems to function as a mediator in the transmissiom of mechanical, thermal and pain impulses in the spinal cord of the pheasant.

\section{CONCLUSION}

In summary, the present study is the first to show the presence of NADPH-d in the thoracic spinal cord of the pheasant, and to compare it with the distribution of NADPH-d positive neurons in the spinal cord of the rat and rabbit. A large number of NADPH-d positive structures appear to be involved in spinal sensory processes and visceral regulation of the rat and rabbit, suggesting that NO may play role of neurotransmitter in the mammalian spinal cord. The distribution of NADPH-d activity in pheasant spinal cord appears to be similar to, but somewhat different from, that in mammals. NO may be involved in many functions in the non-mammalian nervous system including the somatosensory system, but a more exact determination of its role will need further investigations. The difference between mammalian and non- 
mammalian distribution of NADPH-d activity in the spinal cord may reflect an evolutionary aspect of the nitrergic transmission in the nervous system.

\section{REFERENCES}

Anderson C.R., Edwards S.L., Furnes J.B., Bredt B.S., and Snyder S.H.: The distribution of nitric oxide synthase - containing autonomic preganglionic terminals in the rat. Brain Res. 614, 78-85, 1993.

Blottner D., and Baumgarten H.G.: Nitric oxide synthetase (NOS)-containing sympathoadrenal cholinergic neurons of the rat IML-cell column: evidence from histochemistry, immunohistochemistry, and retrograde labeling. J. Comp. Neurol. 316, 45-55, 1992.

Blottner D., Schmidt H.H.H.W., and Baumgarten H.G.: Nitroxergic autonomic neurones in rat spinal cord. NeuroReport 4, 923-926, 1993.

Bredt D.S., and Snyder S.H.: Isolation of nitric oxide synthase, a calmodulin-requiring enzyme. Proc. Natl. Acad. Sci. USA $87,682-685,1990$.

Bredt D.S., and Snyder S.H.: Nitric oxide, a novel neuronal messenger. Neuron 8, 3-11, 1992.

Dawson V.L., Dawson T.M., London E.D., Bredt D.S., and Snyder S.H.: Nitric oxide mediates glutamate neurotoxicity in primary cortical cultures. Proc. Natl. Acad. Sci. USA 88, 6368-6371, 1991.

Dun N.J., Dun S.L., Forstermann U., and Tseng L.F.: Nitric oxide synthase immunoreactivity in rat spinal cord. Neurosci. Lett. 147, 217-220, 1992.

Dun N.J., Dun S.L., Wu S.Y., Forstermann U., Schmidt H.H.H.W., and Tseng L.F.: Nitric oxide synthase immunoreactivity in the rat, mouse, cat and squirrel monkey spinal cord. Neuroscience 54, 845-857, 1993

Funakoshi K., Abe T., and Kishida R.: NADPH-diaphorase activity in the sympathetic preganglionic neurons of the filefish, Stephanolepis cirrhifer. Neurosci. Lett. 191, 181-184, 1995.

Garthwaite J.: Neural nitric oxide signalling. Trends Neurosci $18,51-52,1995$

Grozdanovic Z., Baumgarten H.G., and Bruning G.: Histochemistry of NADPH-diaphorase, a marker for neuronal nitric oxide synthase, in the peripheral autonomic nervous system of the mouse. Neuroscience 48, 225-235, 1992.

Gulati P., Chan A-S., and Leong S-K.: Ultrastructural localisation of NADPH-diaphorase in the chick thymic medulla. Cell Tissue Res. 279, 405-409, 1995.

Hope B.T., and Vincent S.R.: Histochemical characterization of neuronal NADPH diaphorase. J. Histochem. Cytochem. 37, 653-661, 1989

Hope B.T., Michael G.J., Knigge K.M., and Vincent S.R.: Neuronal NADPH diaphorase is a nitric oxide synthase. Proc. Natl. Acad. Sci. USA 88, 2811-2814, 1991.
Jiang P.J., and Terashima S.: Distribution of NADPHdiaphorase in the central nervous system of an infrared-sensitive snake, Trimeresurus flavoviridis. Brain Res. 713, 168 177, 1996.

Johnson I.P.: A quantitative ultrastructural comparison of alpha and gamma motoneurons in the thoracic region of the spinal cord of the adult cat. J. Anat. 147, 55-72, 1986.

Kawatani M., Erdman S.L., and De Groat W.C.: Vasoactive intestinal polypeptide and substance $\mathrm{P}$ in primary afferent pathways to the sacral spinal cord of the cat. J. Comp. Neurol. 241, 327-347, 1985 .

Kiechle F.L., and Malinski T.: Nitric oxide biochemistry, pathophysiology, and detection. Am. J. Clin. Pathol. 100, 567-573, 1993.

Kluchová D., Rybárová S., Schmidtová K., Miklošová M. Lovásová K., and Dorko F.: The effect of occlusion of the abdominal aorta on NADPH-d activity in the rabbit spinal cord. Biologia 54, 227-232, 1999.

Kluchová D., Schmidtová K., Rybárová S., Lovásová K., Pomfy M., Prosbová T., and Vatlak A.: Partial colocalization of NADPH-diaphorase and acetylcholinesterase positivity in spinal cord neurons. Physiol. Res. 49, 151-156, 2000.

Maršala J.: Systematic and functional neuroanatomy. Eds. Osveta, Martin, p. 782, 1985.

Maršala J., Kluchová D., and Maršala M.: Spinal cord gray matter layers rich in NADPH diaphorase positive neurons are refractory to ischemia-reperfusion-induced injury: a histochemical and silver impregnation study in rabbit. Exp. Neurol. $145,165-179,1997$.

Matsumoto T., Nakane M., Pollock J.S., Kuk J.E., and Forstermann U.: A correlation between soluble brain nitric oxide synthase and NADPH-diaphorase activity is only seen after exposure of the tissue to fixative. Neurosci. Lett. 155, 61-64, 1993.

Mizukawa K., Vincent S.R., McGeer P.L., and McGeer E.G. Distribution of reduced nicotinamide adenine dinucleotide phosphate-diaphorase positive cells and fibers in the cat central nervous system. J. Comp. Neurol. 279, 281-311, 1989.

Mizukawa K.: Reduced nicotinamide adenine dinucleotide phosphate-diaphorase histochemistry: Light and electron microscopic investigations. Methods Neurosci. 3, 457-472, 1990

Muňoz M., Muňoz A., Marín O., Alonso J.R., Arevalo R., Porteros A., and Gonzales A.: Topographical distribution of NADPH-diaphorase activity in the central nervous system of the frog, Rana perezi. J. Comp. Neurol. 367, 54-69, 1996.

Necker R.: Specializations in the lumbosacral spinal cord of birds: Morphological and behavioural evidence for a sense of equilibrium. Eur. J. Morphol. 37, 211-214, 1999.

Nemcová V., Petrovicky P., and Donkelaar H.J.: The effect of electrolytic thalamic lesion on the NADPH-diaphorase activity of neurons of the laterodorsal tegmental and pedunculopontine nuclei in rats. J. Chem. Neuroanat. 17, 227-232, 2000. 
O`Brien, C., Woolf C.J., Fitzgerald M., Lindsay R.M., and Molander C.: Differences in the chemical expression of rat primary afferent neurons which innervate skin, muscle or joint. Neuroscience 32, 493-502, 1989.

Pullen A.H., Humphreys P., and Baxter R.G.: Comparative analysis of nitric oxide synthase immunoreactivity in the sacral spinal cord of the cat, macaque and human. J. Anat. 191, 161-175, 1997.

Saito S., Kidd G.J., Trapp B.D., Dawson T.M., Bredt D.S., Wilson D.A., Traystman R.J., Snyder S.H., and Haley D.F.: Rat spinal cord neurons contain nitric oxide synthase. Neuroscience 59, 447-456, 1994.

Scherer-Singler U, Vincent S.R., Kimura $\mathrm{H}$, and McGeer E.G.: Demonstration of a unique population of neurons with NADPH diaphorase histochemistry. J. Neurosci. Methods. 8 229-234, 1983.

Schmidt H.H.H.W., Smith R.M., Nakane M., and Murad F. Ca2+/calmodulin-dependent NO synthase type I: biopteroflavoprotein with $\mathrm{Ca} 2+/$ calmodulin-independen diaphorase and reductase activities. Biochemistry 31, 32433249, 1992.
Valtschanoff J.G., Weinberg R.J., and Rustioni A.: NADPH diaphorase in the spinal cord of rats. J. Comp. Neurol. 321, 209-222, 1992

Vizzard M.A., Erdman S.L., Roppolo J.R., Forsterman U., and DeGroat W.C.: Differential localization of neuronal nitric oxide synthase immunoreactivity and NADPH-diaphorase activity in the cat spinal cord. Cell Tissue Res. 278, 299-309, 1994

Vizzard M.A., Erdman S.L., and De Groat W.C.: The effect of rhizotomy on NADPH diaphorase staining in the lumbar spinal cord of the rat. Brain Res. 607, 349-353, 1993.

Vizzard M.A., Erickson K., and De Groat W.C.: Localization of NADPH diaphorase in the thoracolumbar and sacrococcygeal spinal cord of the dog. J. Auton. Nerv. System 64, 128 142, 1997.

Wu W.: Expression of nitric-oxide synthase (NOS) in injured CNS neurons as shown by NADPH diaphorase histochemistry. Exp. Neurol. 120, 153-159, 1993. 